\title{
Du texte au livre, et retour : la production littéraire à la Renaissance, une création collaborative?
}

\section{Anne Réach-Ngô}

\section{(2) OpenEdition}

\section{Journals}

Édition électronique

URL : http://journals.openedition.org/genesis/1511

DOI : 10.4000/genesis.1511

ISSN : 2268-1590

Éditeur :

Presses universitaires de Paris Sorbonne (PUPS), Société internationale de génétique artistique littéraire et scientifique (SIGALES)

Édition imprimée

Date de publication : 27 novembre 2015

Pagination : $29-47$

ISBN : 9791023105049

ISSN : 1167-5101

\section{Référence électronique}

Anne Réach-Ngô, «Du texte au livre, et retour : la production littéraire à la Renaissance, une création collaborative ? », Genesis [En ligne], 41 | 2015, mis en ligne le 18 avril 2017, consulté le 16 mai 2019. URL : http://journals.openedition.org/genesis/1511; DOI : 10.4000/genesis.1511 


\title{
Du texte au livre, et retour : la production littéraire à la Renaissance, une création collaborative ?
}

Anne Réach-Ngô

\begin{abstract}
$\mathrm{A}$ vec l'invention de l'imprimerie, naît à la Renaissance un nouvel espace, l'atelier de l'imprimeur, où la pensée de l'écrit et celle de sa transmission se voient explorées de concert. Bernard Cerquiglini, dans Éloge de la variante, rappelle que s'y déroulent les premiers rapports de force qui contribueront à la définition de l'auctorialité moderne (fig. 1).

Les études seiziémistes se sont particulièrement intéressées, ces dernières années, à la manière dont ces différents acteurs interviennent dans la conception et la réalisation de l'œuvre littéraire, " mise en belle forme de livre », selon l'expression de Marot ${ }^{1}$. En s'introduisant dans les " arrière-boutiques de la littérature », en examinant le rôle des « passeurs de textes » que sont les gens du livre et hommes de lettres, en posant à nouveaux frais la question de savoir «qui écrit», ces études ont contribué à porter un autre regard sur les pratiques scripturaires de la Renaissance ${ }^{2}$, au point que certaines grandes figures d'auteur, telle Louise Labé, se soient trouvées reléguées au rang de
\end{abstract}

1. Ces recherches n'auraient pu avoir lieu sans les travaux fondateurs de Cynthia J. Brown, et notamment Poets, Patrons and Printers: Crisis of Authority in Late Medieval France, Ithaca, Cornell University Press, 1995. Elles ont également été très nettement inspirées par l'arrivée en France des études anglo-saxonnes relevant de la new bibliography et l'on doit notamment à Henri-Jean Martin et Roger Chartier d'avoir assuré leur application à de nouveaux corpus. Jeanne Veyrin-Forrer a également contribué à leur prise en compte, en France, dans l'analyse du dispositif du livre ancien. Pour une synthèse au sujet de ces questions bibliographiques, voir Daniel Maira, « La bibliographie matérielle renouvelée, entre philologie éditoriale et histoire culturelle », "Paroles dégelées ». Propos de l'Atelier XVIe siècle, Paris, Classiques Garnier, à paraitre.

2. Edwige Keller-Rahbé (dir.), Les Arrière-boutiques de la littérature : auteurs et imprimeurs-libraires aux XVIe et XVII $e^{e}$ siècles, Toulouse, Presses universitaires du Mirail, 2010 ; Christine Bénévent, Annie Charon, Isabelle Diu, Magali Vène (dir.), Passeurs de textes. Imprimeurs et libraires à l'âge de l'humanisme, Paris, Presses de l'École nationale des chartes, 2012 ; Christine Bénévent, Isabelle Diu, Chiara Lastraioli (dir.), Passeurs de textes II : gens du livre et gens de lettres à la Renaissance, Turnhout, Brepols, 2014 ; Martine Furno (dir.), Qui écrit ? Figures de l'auteur et poids des co-élaborateurs du texte, de la fin du manuscrit à la Révolution (XVe-XVIIIe siècles), Lyon, Presses de l'ENS-LSH, 2009 ; Martine Furno, Raphaële Mouren (dir.), Auteur, traducteur, collaborateur, imprimeur... qui écrit? Paris, Classiques Garnier, 2013. Nous nous permettons également de citer les deux premiers ouvrages collectifs que nous avons codirigés, dans le cadre des journées d'étude et colloque organisés par l'Atelier XVIe siècle de Paris-Sorbonne, dont l'étude des «politiques éditoriales » constitue l'un des axes de recherche (http://officinedemercure.org/axes-de-recherche/politiques-editoriales/) : Brigitte Ouvry-Vial, Anne Réach-Ngô (dir.), L'Acte éditorial. Publier à la Renaissance et aujourd' hui, Paris, Classiques Garnier, coll. « Pratiques éditoriales », 2010 ; Anne Arzoumanov, Anne Réach-Ngô, Trung Tran (dir.), Le Discours du livre. Mise en scène du texte et fabrique de l'œuvre sous l'Ancien Régime, Paris, Classiques Garnier, coll. « Pratiques éditoriales », 2011. 


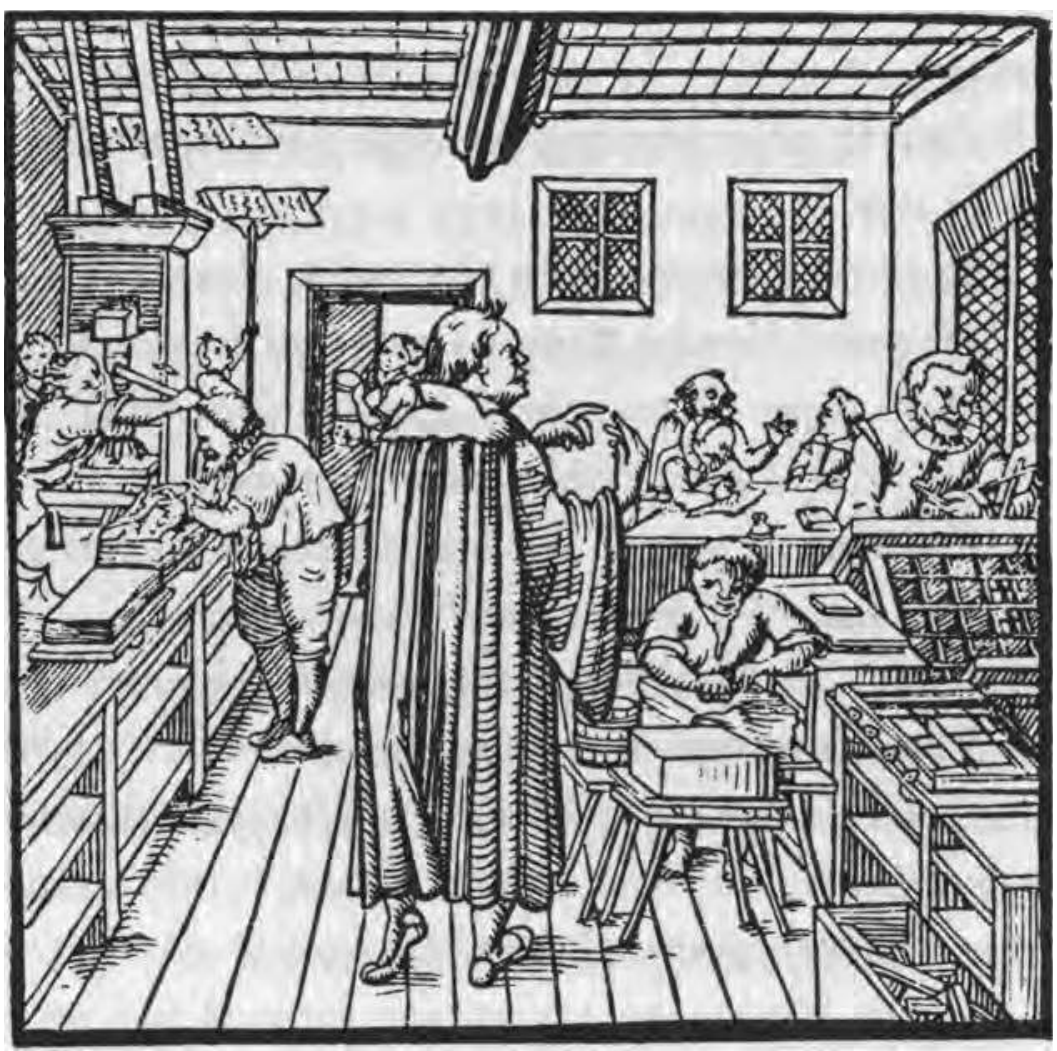

Fig. 1 : Jérôme Hornschuch, Orthotypographia. Instruction utile et nécessaire pour ceux qui vont corriger des livres imprimés et conseils à ceux qui vont les publier, Leipzig, 1608 ; Paris, Éditions des Cendres, 1997

« [...] de dos, massif, au premier plan, organisant l'espace comme il distribue le travail, le maitre imprimeur ; tout autour, affairés et joyeux, les ouvriers de l'Humanisme : tenant la presse, lavant les caractères, séchant la feuille. Une table, au fond, où s'appuie un lecteur placide : le correcteur; enfin, un petit bonhomme s'adresse avec véhémence à quelque contremaître, qui ne l'écoute guère : c'est l'auteur. Un peu ridicule, un peu déplacé, ou du moins qui n'a pas encore trouvé sa place, son statut ${ }^{3}$. »

prête-nom, mais aussi de porte-parole inavoué d'une équipe de rédacteurs masqués, fréquentant assidûment les milieux éditoriaux ${ }^{4}$.

Peut-on pour autant considérer que ces artisans du livre, qualifiés de « co-élaborateurs » par Martine Furno, accèdent au statut de " créateurs », au même titre que le rédacteur du texte, déjà appelé « autheur» ou « acteur », selon les graphies de l'époque ? Le fruit de leur entreprise et la démarche qui y conduit relèvent-ils d'une « création collaborative ${ }^{5}$ » et quels en sont les effets sur l'autorité naissante de l'auteur?

3. Bernard Cerquiglini, Éloge de la variante. Histoire critique de la philologie, Paris, Éditions du Seuil, 1989, p. 21. 4. Mireille Huchon, Louise Labé, une créature de papier, Genève, Droz, coll. « Titre courant », 2006.

5. Nous laisserons ici de côté la question de savoir si l'emploi d'un tel terme pour qualifier la littérature à la Renaissance peut être considéré comme anachronique. Si, selon Olivier Pédeflous, on ne peut en effet parler, à cette époque, de «création » qu'appliquée à l'œuvre divine, on notera que la critique n'a pas hésité à user de ce mot (de l'ouvrage fondateur d'Henri Weber, La Création poétique au XVIe siècle en France de Maurice Scève à Agrippa d'Aubigné, Paris, Nizet, 1955, au collectif récent de Marie-Dominique Legrand et Keith Cameron (dir.), Vocabulaire et création poétique dans les jeunes années de la Pléiade : 1547-1555, Paris, Champion, 2013). Le centre V. L. Saulnier de Paris-Sorbonne s'intitule également « Centre de recherche sur la création littéraire en France à la Renaissance». 
À la Renaissance tout particulièrement, où s'expérimentent les potentialités de ce qui deviendra le livre moderne6, l'acte éditorial ne relève pas, le plus souvent, d'un simple travail d'ornementation, qui succéderait à l'étape achevée de la rédaction d'un écrit ; il s'agit d'une opération qui fait partie intégrante, sinon de la genèse du texte, du moins de la genèse de l'œuvre. Pour envisager le processus de la production littéraire, il convient de bien distinguer les notions de texte, de livre et d'œuvre pour prendre la mesure des jeux d'interaction qui s'opèrent entre ces différentes entités. On désignera par « texte » le produit de l'écriture de l'auteur avant que celui-ci ne soit préparé par le secrétaire ou copiste qui remet au propre cette version initiale et la transmet à l'imprimeur ; par « livre », le résultat de la mutation de cette entité somme toute abstraite (dans la mesure où celui-ci n'a que très rarement été conservé sous sa forme manuscrite) en un objet manufacturé ; et par « œuvre » ce même objet, désormais entendu du point de vue de la réception : le texte mis en livre et destiné à un public. Or les procédés mêmes de fabrication du livre dans l'atelier de l'imprimeur contribuent à brouiller les frontières qui caractérisent ce que l'on considère dans le cas du livre industriel comme trois étapes successives et bien distinctes, que l'on peut attribuer à trois instances clairement identifiables : l'auteur, les acteurs de la chaîne éditoriale, puis les publics et institutions qui concourent à donner un statut d'œuvre au texte préalablement mis en livre.

À la Renaissance, en effet, l'acte de publication - qui n'est pas toujours circonscrit à l'étape de la mise sous presse mais peut prendre de multiples formes, à diverses étapes de l'élaboration de l'œuvre ${ }^{7}$ - sollicite différents acteurs de l'appareil éditorial qui s'attachent à se réapproprier le texte pour en garantir la publiabilité8. Prendre en charge la médiation du texte en assurant sa mutation en objet-livre, convoquer un lectorat susceptible de le recevoir en tant qu'œuvre, remanier le texte pour lui offrir une certaine résistance à l'épreuve du temps, et parfois même, en amont, suggérer implicitement ou explicitement ces considérations au rédacteur premier, futur «auteur », avant même l'entreprise d'écriture, telles sont, rapidement énumérées, les tâches et responsabilités de l'instance éditoriale lors de la fabrication de l'œuvre imprimée, au Xvie siècle.

Les modalités d'une telle participation éditoriale n'ont toutefois pas été examinées en terme de « création » à proprement parler. Forgée dans la lignée des réflexions d'Emmanuël Souchier consacrées à l'« énonciation éditoriale » dans les écrits contemporains', la notion d'« écriture éditoriale » que nous avons élaborée10 présente l'intérêt d'inclure dans cette énonciation les tensions et concurrences qui animent, à la Renaissance, le partage de la paternité littéraire. Née de la rencontre et de l'enchevêtrement de systèmes de signification hétérogènes, typographiques, iconographiques et plastiques, entendus comme autant de

6. Voir à ce sujet l'ouvrage de référence d'Henri-Jean Martin, La Naissance du livre moderne. Mise en page et mise en texte du livre français (XIVe-XVIIe s.), Paris, Éditions du Cercle de la Librairie/Promodis, 2000, et notamment le chapitre intitulé «La normalisation de la prose (XVe-XVII ${ }^{\mathrm{e}}$ siècles) », p. 270-443.

7. Voir les différentes perspectives adoptées dans les nombreuses contributions des ouvrages collectifs précédemment cités.

8. La notion de «publiabilité » est empruntée à Roland Barthes, « Délibération », Tel quel, n 82, 1979 (rééd. dans Le Bruissement de la langue. Essais critiques IV, Paris, Éditions du Seuil, 1984, p. 410-412).

9. Emmanuël Souchier, «L'image du texte. Pour une théorie de l'énonciation éditoriale », Les Cahiers de médiologie, $\mathrm{n}^{\circ} 6,1998$, p. 137-145.

10. Anne Réach-Ngô, L'Écriture éditoriale à la Renaissance. Genèse et promotion du récit sentimental français (1530-1560), Genève, Droz, coll. « Travaux d'Humanisme et Renaissance », 2013.

\section{Quand publier est encore écrire}


« machineries sémantiques 11 », l'œuvre imprimée délivre un discours implicite - verbal (les péritextes) et non verbal (les choix formels) - sur le texte qu'il donne à lire à ses lecteurs. Ce discours hybride comprend bien entendu aussi le discours de l'auteur (le « texte » mis en livre), mais il le met en perspective. Il en propose en somme, tout comme le musicien le fait de la partition, une interprétation.

C'est ainsi en se plaçant du point de vue de la participation de l'instance éditoriale à la création littéraire, plus que de la collaboration engagée avec l'auteur proprement dit, que les premiers jalons d'une réflexion sur la «création collaborative » à la Renaissance ont été jusqu'à présent posés. Cela ne tient pas seulement à des contraintes épistémologiques et méthodologiques : en l'absence quasi totale de «brouillons d'auteur » à disposition, l'entrée dans le processus élaboratif de l'œuvre ne peut se faire, pour l'essentiel, que par l'étude de ses versions déjà imprimées, "brouillons d'éditeur », en quelque sorte. Filtre incontournable pour accéder aux mutations de l'œuvre, le traitement que l'acte de publication fait subir au texte et sa diversité au sein d'éditions simultanées (chez différents imprimeurs) et successives (chez le même imprimeur ou d'un imprimeur à l'autre) conditionne l'entrée du chercheur - par la petite porte, celle de l'atelier d'imprimerie - au sein du processus de la création ${ }^{12}$. Cela impose au chercheur, de manière somme toute très fructueuse, de prendre en considération que la médiation du texte fait partie intégrante de l'élaboration de l'œuvre. Un tel présupposé le conduit alors à examiner la pertinence de la notion de « création collaborative » appliquée à la production littéraire de la Renaissance.

Cette dimension médiatrice est d'autant plus importante qu'elle rend possible la conservation et la transmission à la postérité du contenu du manuscrit auquel le lecteur ne peut avoir accès sans l'intermédiaire éditorial. À ce titre, l'instance éditoriale produit bien, plus encore qu'une interprétation musicale - le connaisseur pouvant se représenter mentalement la composition musicale en lisant la partition -, un dispositif de signification nécessaire à la communication littéraire, lorsque la sociabilité littéraire contemporaine de la production du texte, sa lecture à haute voix ou sa transmission orale, ne sont plus d'actualité. Qualifier d'" écriture » l'intervention des hommes du livre sur le texte qu'ils éditent - tant par sa mise en forme typographique et spatiale que par l'ajout de péritextes revient donc à reconnaître leur pleine participation à la littérarité de l'œuvre. Reste à voir selon quelles modalités la mise en œuvre de cette « écriture éditoriale » relève bien d'une « création collaborative » et ne constitue pas simplement une strate supplémentaire de sens, apposée à la création initiale de l'auteur.

\section{De l'écriture à la publication, et retour}

On l'a dit, la matière même qui s'offre aux chercheurs pour aborder cette question tend sans doute à biaiser l'enquête. Il est rare, en effet, qu'existent suffisamment de documents (correspondances, contrats notariaux entre l'auteur et l'imprimeur, notes d'intention de

11. Roger Chartier, Pratiques de la lecture, « Du livre au lire », Paris, Rivages, 1985, p. 79.

12. Sur la question des enjeux et limites d'une reconstitution génétique sans brouillons, voir notamment Almuth Grésillon et Jean-Louis Lebrave (dir.), Écrire aux XVII et XVIII siècles. Genèses de textes littéraires et philosophiques, Paris, CNRS Éditions, coll. «Textes et Manuscrits », 2000 ; Bernard Beugnot, « La génétique : questions de frontières », dans Olga Anokhina et Sabine Pétillon (dir.), Critique génétique. Concepts, méthodes, outils, Paris, Éditions de l'IMEC, 2009, p. 33-45 ; dir. Patrick Dandrey, Génétique matérielle, génétique virtuelle. Pour une approche généticienne des textes sans archives, Québec, Presses de 1'université de Laval, 2009. 
l'auteur dans d'autres écrits) pour que puissent être dégagés avec précision le partage des tâches et les étapes successives dans l'élaboration de l'œuvre, telle qu'elle se présente in fine sous sa forme imprimée ${ }^{13}$. En revanche, l'importance relative des variantes, textuelles et matérielles, qu'offre l'ensemble des éditions d'une même œuvre constitue un critère efficace pour déceler la part active de l'instance éditoriale dans l'entreprise créatrice, une fois la première édition publiée. Cela revient à déplacer la limite que l'on assigne traditionnellement à la définition de la création littéraire, en considérant que celle-ci ne s'achève pas avec la remise d'un manuscrit à l'imprimeur. Une telle conception, posée en ces termes, serait de toute façon inopérante dans la mesure où la création littéraire à la Renaissance est indissociable de formes de sociabilité intellectuelle (vie des collèges, concours et jeux d'écriture, lectures publiques, circulation manuscrite des textes) qui précèdent et suivent l'acte de publication ${ }^{14}$.

Une telle réserve méthodologique n'est heureusement pas sans intérêt heuristique. Les documents étudiés, avant tout des éditions, témoignent à la fois de l'achèvement du processus de constitution de l'œuvre - le texte, devenu livre, se trouvant dès lors accessible à un public - et de son immédiate remise sur le métier, en un effet de «feed back élaboratif », pourrait-on dire - l'édition publiée étant toujours provisoire, amenée à être reprise, réorganisée, repensée à la lumière des attentes de nouveaux publics cibles, des autres publications qui voient le jour, etc. L'étude des enjeux, manifestations et implications du passage sous la presse (singulier ou répété) d'une même œuvre met alors au jour la dynamique créatrice, non linéaire et à plusieurs mains, qui caractérise la production littéraire à la Renaissance ${ }^{15}$.

L'exemple des mutations éditoriales d'un des premiers récits sentimentaux traduits de l'italien en langue française dans les années 1530, la Flammette de Boccace, en constitue une illustration intéressante ${ }^{16}$. L'appartenance initiale du texte au domaine italien peut sembler constituer un cas bien spécifique dans la production littéraire publiée à la Renaissance, d'autant que l'auteur médiéval, Boccace, est mort depuis plus de cent cinquante ans lorsque paraissent les éditions en langue française de son récit, et que les traducteurs, suivant les conceptions très libres de la translatio à l'époque, ont toute liberté pour modifier le texte et l'adapter à leurs divers publics cibles. En réalité, la dimension « étrangère » de cette littérature ne fait que souligner la part non négligeable des apports indirects, des jeux de circulation et d'emprunts qui conduisent un texte, distinct de son

13. On connaît toutefois quelques exceptions, dont témoigne par exemple la structuration en deux volumes des récents travaux de François Rouget : Ronsard et le livre : étude de critique génétique et d' histoire littéraire. Première partie : lectures et textes manuscrits, Genève, Droz, 2010 ; Seconde partie : Les livres imprimés, Genève, Droz, 2012.

14. Pour une synthèse concernant ces questions, voir l'ouvrage de Richard Crescenzo, Histoire de la littérature française du XVIe siècle, Paris, Champion, 2001 et notamment les chapitres « Société, mondes littéraires, écrivains » et « Production, édition et médiations des œuvres littéraires », p. 7-28 et 29-42.

15. Voir Anne Réach-Ngô (dir.), « Genèses éditoriales », dossier thématique de la revue Seizième Siècle, n 10 , 2014. Ont participé à ce volume : Jean Balsamo, François Rouget, Daniel Maira, Cynthia Brown, Olivier Pédeflous, Mireille Huchon, Grégoire Holtz, Anne-Marie Lievens, Olivier Deloignon, Olivier Grellety Bosviel, Guillaume Berthon et Anne Réach-Ngô.

16. Sur l'histoire éditoriale de ce récit, voir la fiche de la base de données Éditions Lyonnaises de Romans dirigée par Pascale Mounier, sur le site de l'association RHR (Renaissance, Humanisme, Réforme) : <www.rhr16.fr/baseelr/ouvrage/30/\%5BFiammetta\%5D+Flammette>. 


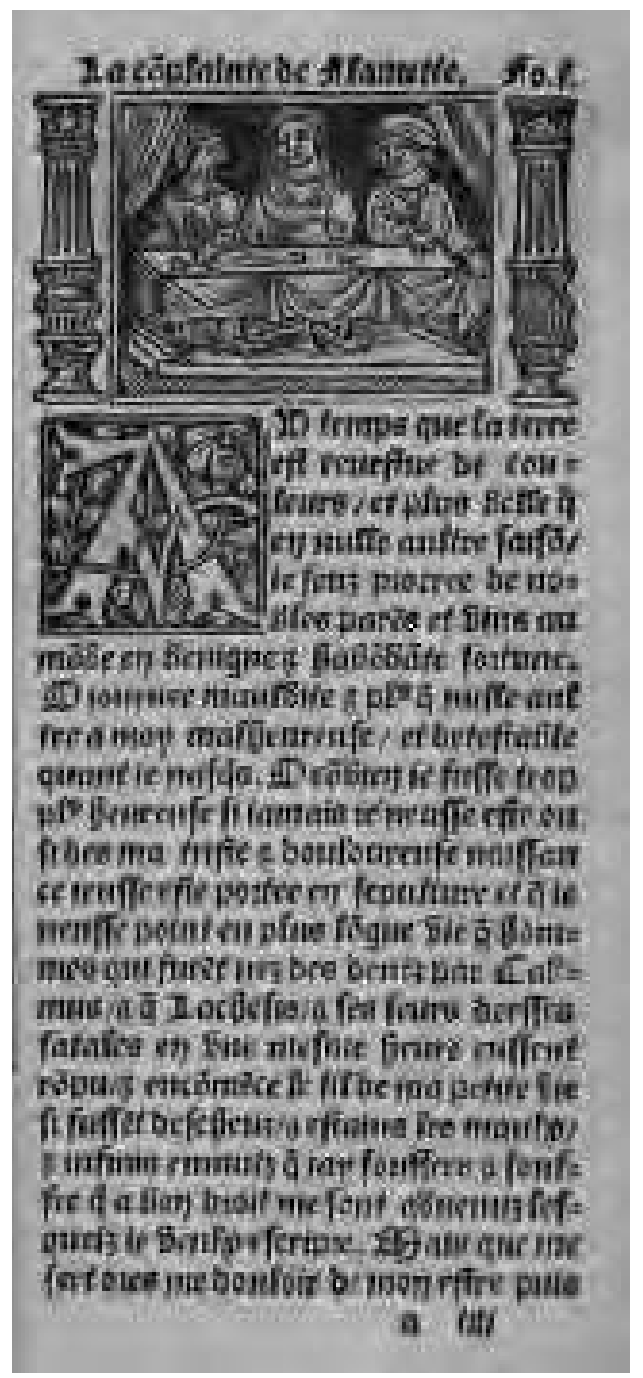

Fig. 2 : Jean Boccace, Complainte tres piteuse de Flamette à son amy Pamphile. Translatée d'Italien en vulgaire francoys, Lyon, François Juste, 1532 (Gordon Collection de la Bibliothèque de l'Université de Virginie, Gordon

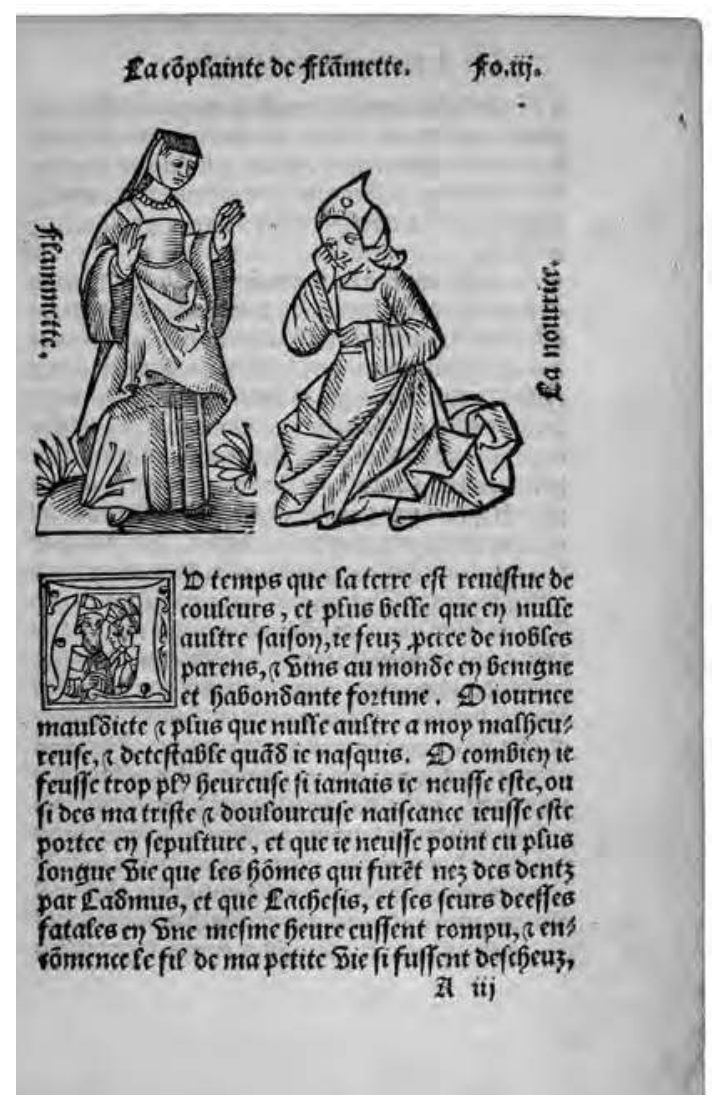

Fig. 3 : Jean Boccace, Flammette. Complainte des tristes amours de Flammette à son amy Pamphile, Translatée d'Italien en vulgaire Francoys, Lyon, Claude Nourry, 1532 (BnF, Rés. Y2-2248, $\mathrm{f}^{\circ}$ iiir $^{\circ}$ ) 1532)

auteur, à un imprimeur qui va prendre en charge sa publication, phénomène très courant à la Renaissance, y compris lorsque l'auteur est vivant.

Le récit de la Flammette connaît au XVI e siècle de nombreuses éditions italiennes, régulièrement rafraîchies et corrigées, publiées notamment à Venise et à Florence. L'étape de l'établissement du texte à partir de la version manuscrite introduit d'emblée une première distance entre le texte issu de la plume de Boccace et celui que l'imprimeur donne à lire à ses lecteurs. L'opération de traduction, de l'italien au français, en constitue une seconde étape qui ouvre la voie à toutes sortes de modifications nouvelles. Les premières éditions conservées datant de 1532 sortent des presses lyonnaises de Claude Nourry et de François Juste sous le titre : Complainte tres piteuse de Flamette à son amy Pamphile. Translatée d'Italien en vulgaire francoys. Dans le même temps, paraît à Paris une édition d'Antoine Bonnemère, Jean Longis et la veuve Jean Saint-Denis, sous le titre 


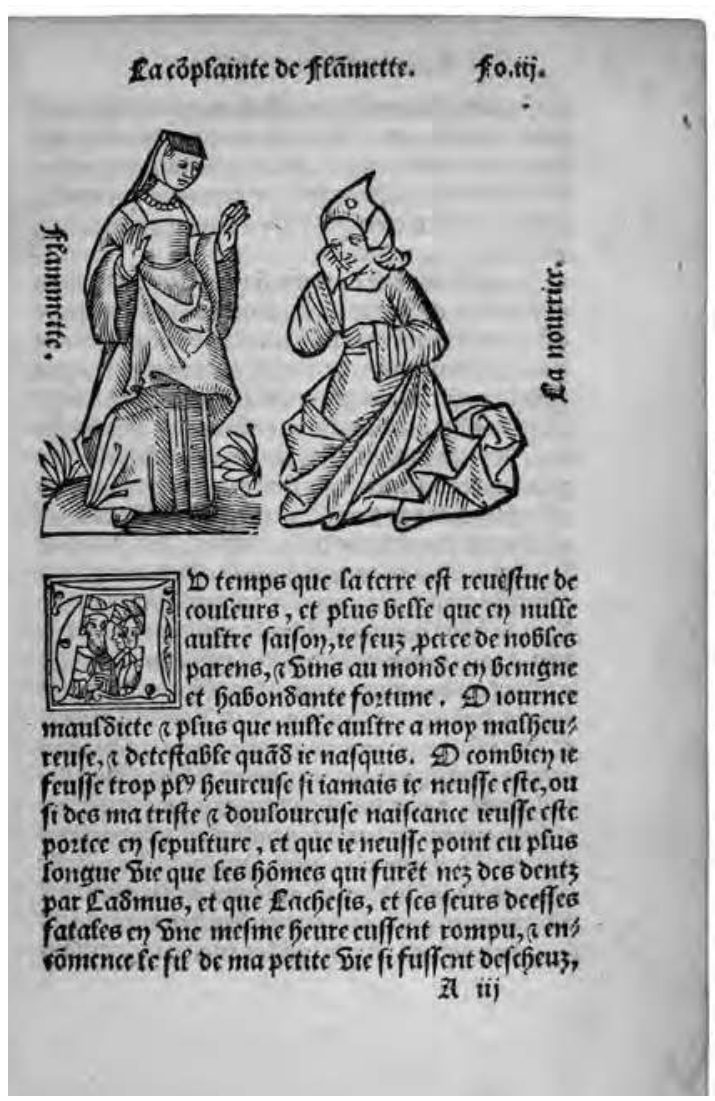

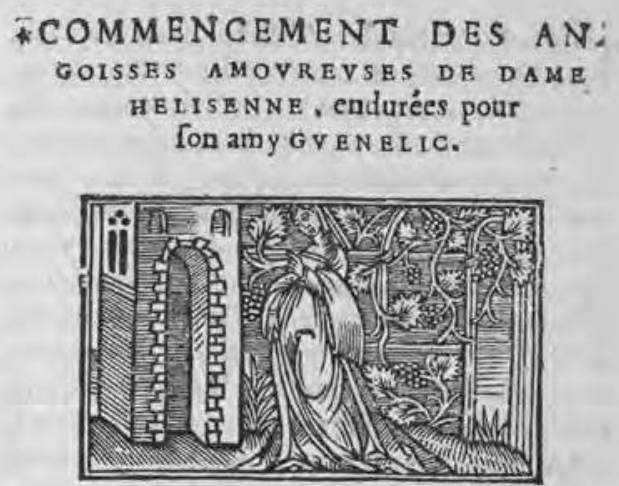

अक्ष temps que la déeffe сг в в $t$ e de. A (2) Ipouilla fon glacial \& gelide habit, 2. 3.1 . I veftit fa verdoy ante robbe tapifféc 20 20 de diuerfes couleurs, ie fuz procrée de nobleffe, \& fuz caufe à ma naiffance, de reduy re en grand ioye \& lyefle mes plus prochains parens, qui font pere \& mere, par ce qu'ilz eftoient hors d'efperánce de iamais auoir generation. 0 qu'a iufte caufe, ie doibs mauldire l'heure que ie nafquis, las que ie fuz née en mauluaife conftellation, Ie crois qu'il n'eftoit Dieu au ciel,ne fortune en terre pour moy. O que ỉeuffe efté heureufe, fi le laict maternel m'euft efté venin, qui euft efté caufe de la tranfmigratió de l'ame, fané ce qu'elle euft efté agitée de tant grand anxiets A iii

Fig. 4 : Hélisenne de Crenne, Les Angoysses douloureuses qui procedent d' amours, Paris, Denis Janot, 1538

(BnF, Rés. P-Z-2013, f ${ }^{\circ}$ Aiii $\mathrm{r}^{\circ}$ )

La Complainte de Flamette à son amy Pamphile. Les éditions se font concurrence17 et offrent différentes interprétations du même texte-source, témoignant par là même de la marge de manœuvre dont dispose l'équipe éditoriale pour transmettre un livre, et non un seul texte, à ses lecteurs (fig. 2 et 3 pour les deux éditions lyonnaises - on notera en particulier l'effet produit par la différence de format de l'édition).

La traduction de Boccace sert ensuite de source, mais aussi de modèle typographique, à un autre récit sentimental, Les Angoysses douloureuses qui procedent d'amours, attribué à Hélisenne de Crenne, le premier à paraître en langue française sans être le produit d'une traduction, même si la collecte de nombreux morceaux empruntés à toutes sortes de récits étrangers témoignent d'un brassage linguistique et d'une circulation des textes d'un pays à l'autre18. L'emprunt au livre de la Flammette, et non au seul texte, est manifeste dès l'incipit (comparer les fig. 3 et 4 ) et souligne la portée de la forme dans la citation intertextuelle de l'ouvrage.

17. Voir notamment l'article de William Kemp, «Les éditions parisiennes et lyonnaises de la Complainte de Flammette de Boccace (1531-1541) », Studi Francesi, XCVIII, 1989, p. 247-265.

18. L'ouvrage est paru chez Denis Janot en 1538 . Nous avons comparé les variantes des différentes éditions, parues entre Paris et Lyon, de 1538 à 1560, pour en décomposer la genèse, fort complexe. Nous nous permettons de renvoyer ici à notre ouvrage : Anne Réach-Ngô, L'Écriture éditoriale à la Renaissance, op. cit. 
C'est un même modèle typographique qui est repris à l'ouverture de l'œuvre, avec des déplacement d'accent, notamment en matière iconographique : à une représentation traditionnelle de l'héroïne accompagnée de sa nourrice se substitue une figure féminine solitaire, dans un verger, qui renvoie à un autre imaginaire narratif et tend à recentrer l'histoire sur l'intériorité du narrateur-personnage.

L'influence du succès de ce livre, dont Denis Janot a assuré la promotion à Paris en 1538, conduit ce même imprimeur à rééditer le texte boccacien en 1541, lui appliquant les critères de mise en forme élaborés pour l'édition des Angoysses douloureuses parue trois ans auparavant. Ainsi le texte-source, remanié dans le cadre d'un emprunt intertextuel, se trouve repensé à la lumière des publications environnantes et intégré à une nouvelle famille de livres, suivant les critères de présentation des ouvrages publiés par Denis Janot. Les stratégies éditoriales de l'imprimeur concourent alors à lui donner une tonalité nouvelle, liée à l'actualité du genre sentimental en plein déploiement au cours des années 1530. Si le texte initial de Boccace demeure pour l'essentiel inchangé, l'œuvre que le lecteur reçoit est tout autre, et sa parenté avec de nouveaux livres à succès devient l'une de ses nouvelles caractéristiques ${ }^{19}$.

Passée cette actualité qui tient à l'émergence du genre sentimental, le récit se prête à de nouvelles modifications éditoriales qui affectent sa signification, de même que les protocoles de lecture suggérés aux lecteurs. Ainsi la parution de l'ouvrage en une édition bilingue destinée à ceux qui souhaitent apprendre l'italien par les textes (fig. 5, 6 et 7) contribue, à la fin du siècle, à redonner une identité italienne à un récit qui avait été importé dans l'univers de référence culturel français au cours des années 1530-1540. Les modifications textuelles visent également à en faciliter l'utilisation suivant une perspective pédagogique.

Dans cette version bilingue, le texte et sa traduction se trouvent tous deux simplifiés et notamment la syntaxe particulièrement sinueuse du texte boccacien, clairement élaguée. C'est une nouvelle «Fiammette amoureuse » qui se trouve offerte aux lecteurs, une forme d'adaptation prise en charge par Gabriel Chappuys.

Difficile de ne pas voir dans cette circulation, allant de l'écriture à la publication et de la publication à l'écriture, une parenté frappante avec le développement, de nos jours, de l'édition électronique et de l'écriture collaborative en ligne. Dans un cas comme dans l'autre, la répartition des responsabilités et des tâches, qui, concernant le manuscrit médiéval comme le livre industriel, reposait sur un ensemble d'étapes successives clairement définies, gagne en complexité et laisse place à l'innovation. Sans doute cela tient-il à la fécondité des périodes de révolution médiologique, la nouveauté des outils à disposition suscitant chez les différents corps de métier le désir d'en explorer les potentialités en donnant naissance à des initiatives qui favorisent les collaborations inédites. La disparition des éditions précédentes et la faible diffusion des tirages dont l'impact reste local et chronologiquement restreint contribuent à faire du livre dernièrement paru celui qui vaut pour référence, voire pour identité de l'œuvre. Le parallèle conforte en tout cas l'hypothèse de l'existence d'une création collaborative entre milieux lettrés et milieux éditoriaux, à la Renaissance.

19. Sur cette question, nous renvoyons à l'article de Michèle Clément, "Co-élaborations à Lyon entre 1532 et 1542 : des interventions lyonnaises en réseau sur les "récits sentimentaux"? », RHR, n 71, décembre 2010, p. 35-44. Notre analyse, quant à elle, se concentre sur la dimension typographique de ce réseau. 

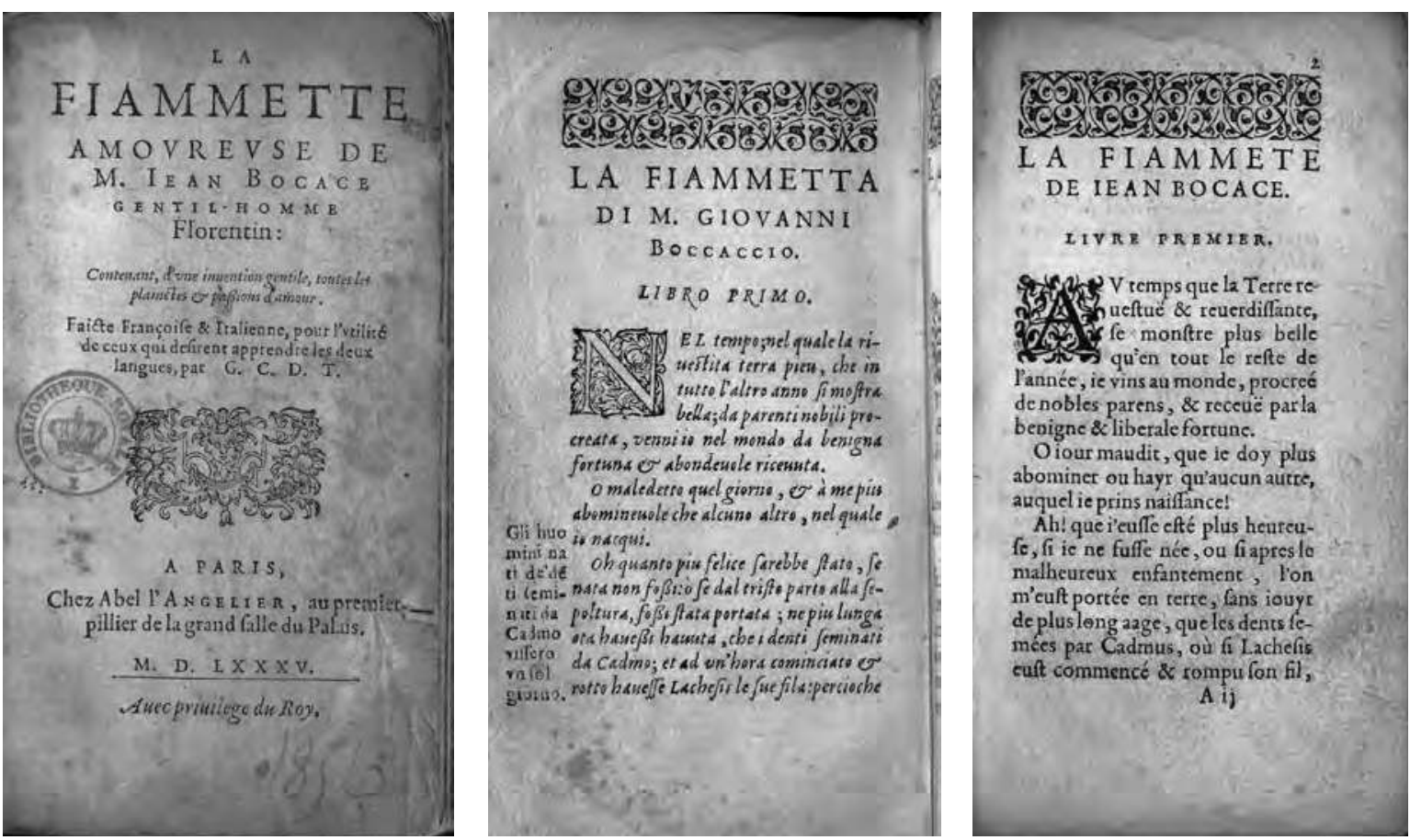

Fig. 5, 6 et 7 : La Fiammette amoureuse de M. Jean Boccace gentilhomme florentin : contenant, d'une invention gentile, toutes les plainctes et passions d'amour. Faicte françoise et italienne pour l'utilité de ceux qui désirent apprendre les deux langues par G. C. D. T. [Gabriel Chappuis, de Tours], Paris, Abel L'Angelier, 1585

(BnF, Rés. Y2-18513, p. de titre, p. [1] et p. 2 (Aii)

Les reconfigurations engagées par l'apparition et le développement de l'imprimerie conduisent alors à poser la question de la paternité littéraire et de l'autorité de l'auteur en de nouveaux termes. Alors que la majorité des manuscrits médiévaux relevait d'auteurs restés anonymes, l'émergence d'une entité auctoriale assure une identité nouvelle à l'œuvre littéraire, désormais caractérisée non seulement par un titre, mais aussi par un nom d'auteur. L'apparition de la page de titre n'est pas étrangère à un tel processus, espace où s'affiche le lien entre l'identification de l'œuvre et le nom de son auteur. Mais un tel espace scriptographique constitue également un lieu de signatures multiples (nom du traducteur, marque de l'imprimeur, adresse du libraire, mention éventuelle d'un privilège royal ${ }^{20}$. Aussi la page de titre représente-t-elle symboliquement les collaborations en jeu dans l'atelier de l'imprimeur, quand cela ne l'est pas figurativement, comme sur la marque d'imprimeur de Josse Bade par exemple, qui représente, en tête de l'œuvre, une presse et l'atelier d'imprimerie en arrière-plan (fig. 8). On le voit bien, le dispositif de présentation de l'imprimeur s'affirme très nettement, comme si celui-ci se faisait le porte-parole du texte, dont le nom de l'auteur n'apparaît que fort discrètement, en petits caractères noirs.

Si la signature des différents participants n'est pas toujours aussi manifeste, l'œuvre imprimée est bien le fruit d'une entreprise à plusieurs mains, et ce, selon les divers postes d'intervention et compétences. Plus encore qu'une création collaborative, la réalisation

\section{La paternité partagée du livre ou l'auteur dépossédé ?}

20. Sur cette question, nous nous permettons de renvoyer à notre article, « Partage de l'autorité et anonymisation de l'œuvre : patronymes et signatures dans l'œuvre imprimé de François Patrice de Sienne », Littératures classiques, $\mathrm{n}^{\circ}$ 80, « L'anonymat de l'œuvre (XVI ${ }^{\mathrm{e}}$-XVIII') $)$, 2013, p. 19-33. 


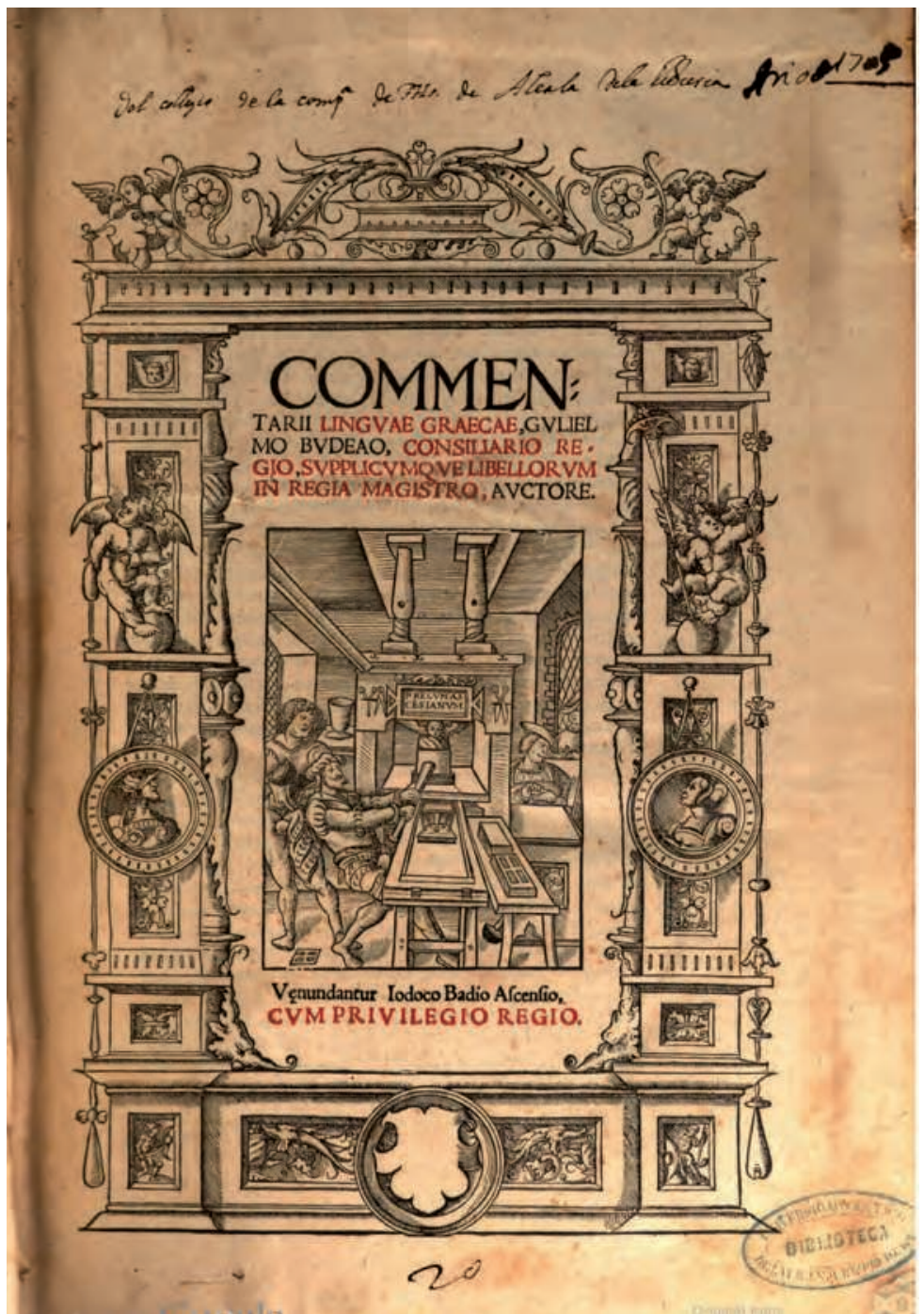

Fig. 8 : Page de titre des Commentarii linguae graecae de Guillaume Budé, Paris, Josse Bade, [1529] (Hathi Trust Digital Library)

de l'œuvre relève à la Renaissance d'une "création d'atelier 21 ». "Petites mains » chargées d'assembler les caractères, de corriger le texte, de le traduire, afin de l'adresser à un public historiquement déterminé22 ; « composition à plusieurs mains » lors des fréquents phénomènes de mise en recueil éditoriale 23 ; situations hybrides où l'éditeur,

21. Voir Anne Réach-Ngô (dir.), Créations d'atelier. L'éditeur et la fabrique de l'œuvre à la Renaissance, actes du colloque international de l'Atelier XVI ${ }^{\mathrm{e}}$ siècle (Université Paris-Sorbonne, 31 mai-2 juin 2012), Paris, Classiques Garnier, 2014.

22. Voir les articles de Nora Viet, Diane Desrosiers, Christine Bénévent, Martine Furno et Jean-François Gilmont dans le volume précédemment cité.

23. Voir les articles de Nathalie Hervé, Anne Réach-Ngô et Louise Amazan. 
auteur ou musicien, « met la main à l'ouvrage24 »; phénomènes de « manipulation éditoriale » où la représentation de l'auteur, voire son identité, s'élaborent dans l'atelier de l'imprimeur25 : autant de cas de figure qui témoignent de l'enchevêtrement des mains dont est nécessairement issue l'œuvre imprimée à la Renaissance. Or de la main de l'auteur, on n'a conservé que peu de traces au sein du livre imprimé, tout au plus de rares cas de corrections d'auteur, tandis que le geste éditorial, né de ces multiples autres mains, demeure inscrit au sein de la matière même de l'œuvre, dans le choix du caractère typographique, de l'illustration comme de la mise en espace du texte. Le discours auctorial lui-même, souvent présent dans des préfaces et avis au lecteur, s'insère au sein d'un dispositif péritextuel complexe, pris en charge, in fine, par l'équipe éditoriale.

Il est un auteur de la fin du Xvie siècle, Étienne Tabourot, qui, dans ses Bigarrures, montre qu'il a parfaitement compris ces divers rouages de la production littéraire, et va, pour ne pas les subir, les mettre en scène, nous donnant à voir de manière plus ou moins fictive l'éventail des modalités d'intervention éditoriale en œuvre à la Renaissance. Comme il le déclare lui-même dès la première édition des Bigarrures du Seigneur des Accordz, chez Jean Richer en 1583, la publication, qui plus est anonyme, est une mise à l'épreuve, qui peut s'accompagner de critiques, mais également de réappropriations incontrôlées :

Or en un mot je fais declaration que je mets ce livre hors de ma maison et l'expose en public, selon la loy de ceux qui vont en masque, sçavoir pour recepvoir patiemment tous brocards, injures et risées, sans replicquer ny me faire cognoistre. T'asseurant de ma part que je ne treuveray point estrange si quelqu'un faigne prendre la peine de taxe et reprendre mes escrits : Veu que c'est et doibt estre un hazard commun à tous ceux qui mettent leurs œuvres en lumiere [= publier $]^{26}$.

En acceptant de courir un temps le risque de la publication anonyme, dissociant le lien qui commence à unir plus fermement, en ce dernier quart de Xvie siècle, l'œuvre à son auteur, Tabourot affirme ouvrir la voie à toutes sortes de manipulations de l'œuvre, au nom de l'intérêt premier des lecteurs. La preuve réside notamment dans la présence, après la préface de l'auteur, d'un avis au lecteur signé d'un personnage nommé André Pasquet, qui semble, à le lire, prendre en charge l'édition, évoquant la légitimité des pratiques de réappropriation qui passent outre la volonté de l'auteur, même de son vivant :

Il y a environ quatre ans qu'il tumba entre mes mains une partie de ce livre intitulé Bigarrures que l'on envoyait à Paris pour imprimer, duquel je fis faire à la haste une copie assez mal escripte et encore plus mal orthographiée presageant à peu prez ce qui est advenu despuis d'icelluy : Car il fut aussi tost retiré par l'autheur sous une honneste excuse qu'il y vouloit changer, adjouster et diminuer, encor que ce fut comme j'ay certainement cogneu pour en frustrer le public et en joyr seul en sa maison, car despuis il n'en ha jamais escrit sinon par deffaictes et longueurs affectées, jusques dernierement qu'il declara au libraire tout ouvertement, que l'eage, le temps et sa profession luy avoyent faict changer d'humeur et de volunté et qu'il luy seroit mal seant d'advouer ce qu'il avoit fait en ses premiers ans et verdeurs de follastre jeunesse ayant à grand peine accomply dixhuict ans, et qu'après qu'il auroit donné preuve de sa suffisance en quelque brave et docte subjet il advi-

24. Voir les articles de Gaëlle Burg, Nahéma Khattabi et Djurdja Sinko-Depierris.

25. Voir les articles de Christine de Buzon et William Kemp, Gilles Polizzi, Mireille Huchon et Jean Vignes.

26. Étienne Tabourot, Les Bigarrures du Seigneur des Accordz, Paris, Jean Richer, 1583, fo ã 4 rº. 
seroit de ne point estouffer ses petits enfans naturels et illegitimes conceus hors mariage [...]. De sorte que j'ay cogneu appertement que c'estoit une excuse recherchée pour nous entretenir, qui m'a occasionné de mettre en lumiere, ce que j'en avois de copie avec des libres adjonctions $[\ldots]^{27}$.

Ce discours suggère ainsi que la volonté éditoriale de satisfaire la demande du lectorat l'emporterait sur les intentions de l'auteur.

Ces pratiques devaient être courantes, en effet, au XVI $\mathrm{I}^{\mathrm{e}}$ siècle, comme en témoignent les préfaces bien connues des œuvres marotiques rendant compte des échanges du poète avec ses imprimeurs 28 . Toutefois, dans le cas de Tabourot, l'auteur ne semble pas complètement étranger à ces stratégies : selon Francis Goyet, en effet, André Pasquet, qui demeure inconnu, pourrait bien être une invention de l'auteur 29 . Le dédoublement des préfaces entre auteur et éditeur relèverait alors d'un jeu auctorial visant à dénoncer, mais aussi à se réapproprier à son tour, des pratiques éditoriales douteuses, et par ailleurs avérées. S'inscrivant dans le topos du manuscrit trouvé, envisagé d'un point de vue éditorial, l'auteur dénonce dès lors les procédés d'intervention éditoriale dont usent à l'époque les imprimeurs.

C'est le cas de l'utilisation qui est faite du portrait d'auteur qui figure au verso de la page de titre, associé à sa devise «A tous accords ». En regard de ce portrait, une strophe de l'auteur souligne, non sans malice, la dimension peu référentielle d'une telle illustration, opposant au grand âge de la représentation le jeune âge de l'auteur des pièces rassemblées ${ }^{30}$. Ce dispositif est repris dans l'édition de 1595 que nous reproduisons ici (fig. 9, 10 et 11) :

27. Ibid., fos ã $6 \mathrm{r}^{\circ}-\tilde{a} 7 \mathrm{r}^{\circ}$.

28. Citons à titre d'exemple la fameuse formule de Marot : «Je ne scay (mes treschers freres), qui m'a plus incité à mettre ces miennes petites jeunesses en lumiere, ou voz continuelles prieres, ou le desplaisir que j'ay eu d'en ouyr cryer et publier par les rues une grande partie toute incorrecte, mal imprimee, et plus au prouffit du libraire, qu'a l'honneur de L'Autheur» (C. Marot, Adolescence clémentine, Paris, Pierre Roffet, 1534, p. 3). Voir notamment à ce sujet l'article de Philippe Desan, " "Profit du libraire" et "honneur de l'auteur" : Marot face à ses lecteurs », Clément Marot « prince des poëtes françois », 1496-1996, Paris, Champion, 1997, p. 683-697.

29. Nous renvoyons pour l'analyse détaillée de l'ouvrage à l'introduction de l'édition critique de Françis Goyet : Estienne Tabourot, Les Bigarrures du Seigneur des Accords, Genève, Droz, coll. « Textes littéraires français », 1986. 30. Le portrait de l'auteur est suivi du quatrain suivant : «Quiconque voit icy le Seigneur des Accords, / Encore qu'il ne soit pas naïvement pourtraict, / Qu'il juge seulement à voir le simple traict, / Qu'il est entier et rond dedans, comme dehors », tandis que sur la page de droite figure la strophe suivante : «L'Imprimeur qui voulut faire une Bigarrure, / Mit un visage tel que je porte à cet'heure / Au front de ce livret, que fort jeune je fis : / Mais il la doit garder pour mes autres escrits, / Et s'il veut mettre icy proprement ma peinture. / Je lui envoyeray lors que j'auray le poil gris. » Cette mise en regard des deux portraits, par la gravure et par le texte de l'auteur, peut évoquer cette confession de Montaigne justifiant sa pratique de l'augmentation et non de la correction : « Mes premieres publications furent l'an mille cinq cens quatre vingts. Depuis d'un long traict de temps je suis envieilli, mais assagi je ne le suis certes pas d'un pouce. Moy à cette heure et moy tantost sommes bien deux ; mais quand meilleur, je n'en puis rien dire. Il feroit beau estre vieil si nous ne marchions que vers l'amendement. C'est un mouvement d'yvroigne titubant, vertigineux, informe, ou des joncs que l'air manie casuellement selon soy » (édition en ligne : <www.lib. uchicago.edu/efts/ARTFL/projects/montaigne/essais.3.html>, § 964). 

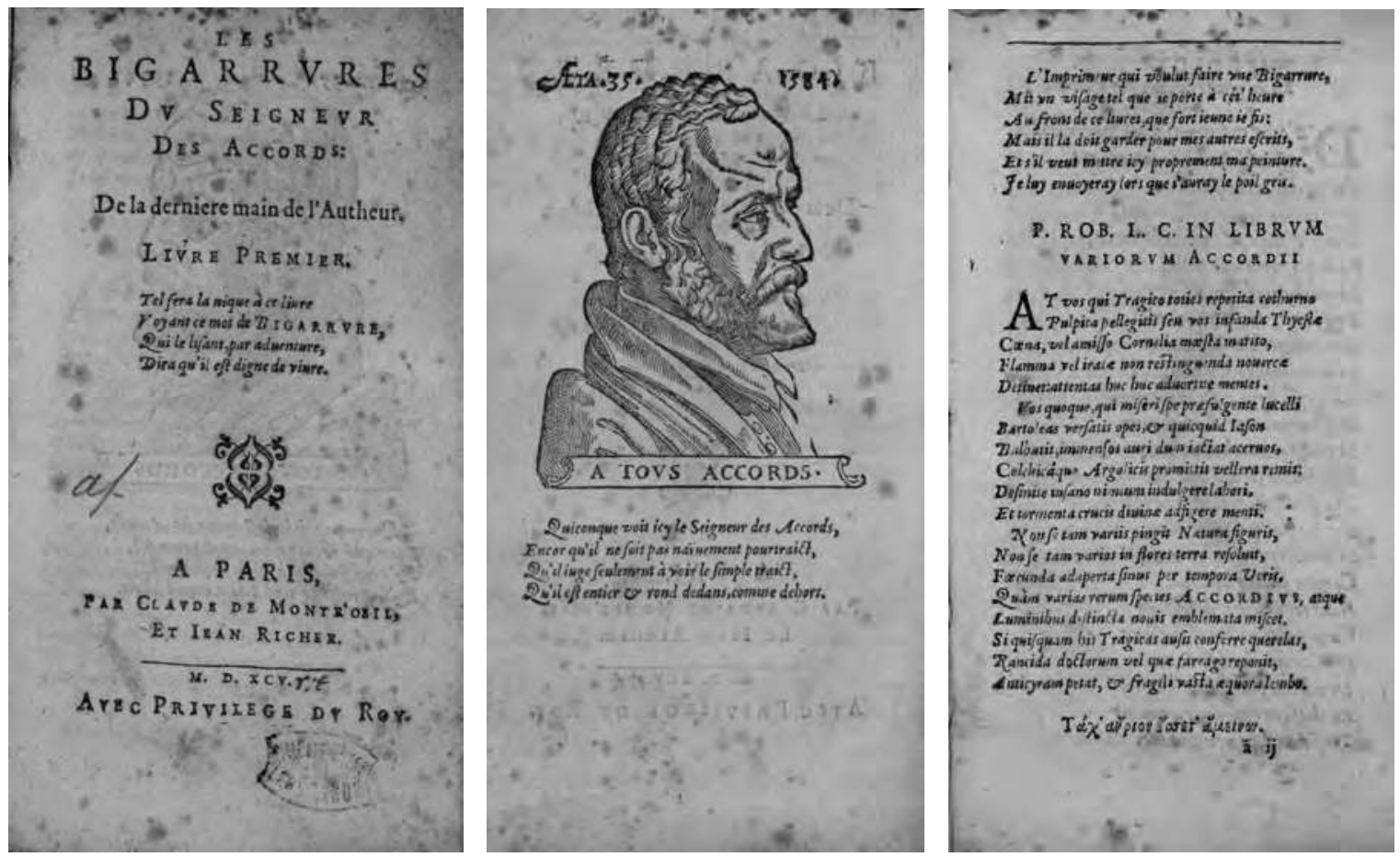

Fig. 9, 10 et 11 : Étienne Tabourot des Accords, Les Bigarrures du seigneur des Accords, Paris, Claude de Montr'œil et Jean Richer, 1595 , page de titre, verso de la page de titre, $\mathrm{f}^{\circ} \tilde{a} 2 \mathrm{r}^{\circ}$

(Bibliothèques virtuelles humanistes, CESR, Tours)

(C) Médiathèque François Mitterrand (Poitiers)

Tabourot, tout comme Montaigne soucieux de préserver au sein de ses Essais les multiples moi qui ont contribué à son écriture, différencie ainsi le jeune poète qu'il a été de celui qui se retourne rétrospectivement vers ses écrits et les donne à publier une dernière fois. Il rappelle ainsi combien la publication propose une superposition artificielle des écrits et des représentations auctoriales, notamment lorsque l'augmentation est au principe de la genèse du volume.

L'« Avant-propos de l'Autheur sur les impressions de ce livre » qui apparait dans une édition ultérieure, insiste sur ses strates successives d'écriture que l'acte de publication transforme à chaque publication, au point que, le temps passant, l'auteur ne reconnaît pas son propre ouvrage lorsqu'il le rencontre à nouveau :

Je fus fort estonné quand je vy la premiere impression de ce livre, duquel je pensois que la mémoire fust esteinte. Mais le relisant, quasi comme chose nouvelle, que je n'avoy veu y avoit quatorze ans, je recognu incontinent et mon genie, et mon style du temps que je l'avois basty, pour me chatoüiller moy-mesme, à fin de me faire rire le premier, et puis apres les autres : tellement que je n'avois observé autre ordre, sinon d'entasser pesle-mesle les exemples, selon qu'ils me venoyent en fantasie. N'estant ce livre que pieces rapportees, sans aucune curiosité, et fait seulement par petits papiers, à diverses fois adjoustez desquels je recognu toutefois qu'une grand'partie avoit esté perdue 31 .

31. Pour des questions de commodité, nous citons ce passage à partir de l'édition de 1595, mais le texte apparaît bien avant : Étienne Tabourot des Accords, Les Bigarrures du seigneur des Accords, Paris, Claude de Montr'œil et Jean Richer, 1595, fos ã $3 v^{\circ}$ - ã $4 r^{\circ}$. 
À ce qu'il dit, c'est finalement l'incomplétude du volume qui pousse Tabourot à intervenir et à produire lui-même de nouvelles pièces, afin que son imprimeur fasse parâtre un recueil à jour. L'acte de publication constitue alors un moteur à l'augmentation et à la remise de l'ouvrage sur le métier. Dans la saynette qu'il donne à lire de son nouveau projet de publication, Tabourot s'amuse ainsi une nouvelle fois à dépeindre comment son œuvre lui a échappé :

Tellement comme chacun est amateur de son ouvrage, je me deliberay lors d'envoyer le surplus des Adjonctions, qui estoient creuës depuis ce temps là, avec celles que l'on avoit obmises. Mais le malheur a voulu que l'Imprimeur, auquel je l'avois envoyé par ces petits paperats, ne les a pas tous receu : ou, comme je croy, les mit entre les mains de quelqu'un, que j'eusse bien voulu ne m'estre pas si familier en cest endroit, lequel les a retranché, et au lieu d'icelles, y a subrogé des Adjonctions de son style, si peu correspondantes au mien, et esloignees de ma conception, qu'il est aisé à voir, à quiconque aura tant soit peu de jugement, que cela n'est du mesme autheur ${ }^{32}$.

Le constat de ces transformations ne provoque pas chez Tabourot un véritable sentiment d'indignation et de dépossession ; l'auteur accepte les ajouts de l'« augmenteur » et en reconnaît la valeur. Les quelques exemples d'adjonctions, selon lui, en effet

[...] montstrent assez la diversité de mon stile au sien, et toutesfois je ne nieray pas qu'ils ne soyent, peut estre, au goust de quelques uns, aussi bons que les miens. Tellement que pour ceste raison, et comme aussi j'ay sceu que c'est quelque docte personnage, qui n'a point fait de malice ces Adjonctions, je ne les ay pas voulu oster nu m'estomaquer à l'encontre de luy : Je me contente de le prier, et tous autres, à l'advenir, d'estre plus religieux à traicter les escrits d'autruy 33 .

Tabourot suggère alors lui-même une autre forme de présentation, consistant à placer à la fin de chaque chapitre une section intitulée " adjonctions », car "Il est bon que l'autheur soit tousjours semblable à soy, et ne me plais point qu'on me donne les choses d'autruy pour les miennes, comme aussi je ne veux pas qu'aucuns s'empare de ce qui est moy ${ }^{34}$ ». Toutefois, le travail de redistribution que nécessite une telle mise à jour entraine à son tour de nouvelles modifications auctoriales :

Occasion dequoy j'ay releu ce folastre livre de bout à autre, ce que jamais auparavant je n'avois fait, à fin de le remettre en lumiere, selon ma vraye conception. Et pource que depuis ce temps-là quelques petites curiositez me sont venues en mémoire, et autres m'ont esté amiablement envoyées par un des plus doctes de nostre France, sur le mesme subjet, je les ay adjousté par forme d'Adjonction de l'autheur, à fin que l'on voye mesme à present, si je suis dissemblable à celuy que j'estais alors, et, qu'à mon exemple, ceux qui voudront faire des Adjonctions, les mettent en ceste façon 35 .

Le discours d'Étienne Tabourot, tout au long de cet avant-propos, rassemble donc de manière parodique bon nombre des enjeux propres à la création collaborative dans l'atelier de l'imprimeur : de multiples mains, identifiées et anonymes, différentes campagnes de

\footnotetext{
32. Ibid., fo ã $4 \mathrm{r}^{\circ}$.

33. Ibid., fo ã $5 \mathrm{v}^{\circ}$.

34. Ibid., fo ã $5 \mathrm{r}^{\circ}$.

35. Ibid.
} 
rédaction, l'importance accordée à l'agencement du volume et aux péritextes, et surtout le jeu des représentations qui préside à l'inscription de ces multiples signatures au sein du volume. En mentionnant ces situations diverses, sans doute tirées d'expériences plus ou moins vécues, l'auteur illustre, à travers un détour par la mise en scène fictionnelle, la diversité de ces manipulations éditoriales que le texte de la Renaissance est à même de subir.

Par ce jeu parodique, Tabourot se démarque ainsi de tout un ensemble d'auteurs qui ont vu leur nom mêlé à ceux des autres sans que le souci de l'auteur n'ait été pris en compte dans l'entreprise éditoriale. Est-ce à dire que la démultiplication de telles signatures interdit l'expression progressive d'une autorité d'auteur, ou qu'elle la dilue, au nom d'un partage de la paternité hautement revendiquée par les instances éditoriales ? Pour notre part, nous pensons plutôt que les traces d'une telle collaboration, en matière de création littéraire, sont, délibérément, tantôt masquées, tantôt surexposées comme le fait Tabourot, pour mettre en scène une forme nouvelle d'auctorialité36. Quelles représentations de l'autorité de l'auteur émanent donc d'un tel processus de création collaborative et comment celle-ci trouve-t-elle à s'inscrire au sein de l'espace du livre ?

Intégré au sein d'une équipe de gens de lettres dont le nom n'apparaît pas toujours sur la page de titre (correcteur, éventuel remanieur, etc.) ${ }^{37}$, associé à des artisans dont la pratique ne relève pas des arts libéraux (compositeur, dessinateur, graveur, éventuellement relieur) ${ }^{38}$, l'auteur de la Renaissance se différencie bien de ses collaborateurs par l'entreprise scripturaire qui le caractérise ${ }^{39}$. Le traducteur, à cet égard, se rapproche bien davantage de sa sphère d'autorité auctoriale ${ }^{40}$, voire s'y substitue partiellement ${ }^{41}$. Citons à cet égard l'éloge de Belleforest que dresse François d'Amboise, traducteur des Heures de recreation et apres-disnées de Guicciardin :

Tu nous traduis icy, ô premier de la France!

O grand Belleforest ! tu traduis Guicciardin,

36. Sur la mise en scène des rapports de force et des conflits entre auteurs et imprimeurs à la Renaissance, voir notre article, «Les représentations polémiques de la paternité littéraire au seuil des livres imprimés de la Renaissance : règlement de compte ou consensus publicitaire ? », dans Pascale Hummel (dir.), Mélivres, Paris, Philologium, 2009, p. 13-29. L'inscription d'une figure d'auteur promue au sein de l'espace du livre dans l'atelier de l'imprimeur a été étudiée dans Anne Réach-Ngô, «Instances et stratégies éditoriales à la Renaissance : de la fabrique du livre à la fabrication de l'auteur », dir. Marie-Pier Luneau et Josée Vincent, La Fabrication de l'auteur, Québec, Nota Bene, 2010, p. 333-362.

37. Voir Martine Furno, Qui écrit ?, op. cit.

38. Voir Jeanne Veyrin-Forrer, « Fabriquer un livre au XVI ${ }^{\mathrm{e}}$ siècle », dans Roger Chartier et Henri-Jean Martin (dir.), Histoire de l'édition française, t. I, « Le livre conquérant. Du Moyen Âge au milieu du XVII siècle », Paris, Promodis/ Le Cercle de la Librairie, 1982, p. 279-301.

39. Voir, à titre d'exemples, les enjeux soulevés par Lise Sabourin (dir.), Le Statut littéraire de l'écrivain, dénomination, condition, représentation, Boulogne, ADIREL, « Travaux de littérature », vol. XX, 2007.

40. Voir à ce sujet la thèse de Mathilde Thorel, «Langue "translative" et fiction sentimentale (1525-1540) : renouvellement générique et stylistique de la prose narrative », thèse dactylographiée, Univ. Jean-Moulin-Lyon III, 2006. 41. On pense par exemple à la saga chevaleresque des Amadis de Gaule qui, d'une traduction espagnole d'un texte de Montalvo, finit par devenir, au fil de l'augmentation des livres, un véritable miroir de la société française.

\section{Du partage de \\ la paternité à la promotion d'une autorité de l'auteur}


Guicciardin dont se vante et le vieill Apennin, Et l'Arne Ethrurien, et toute la Florence.

Toy premier Capitaine a dompter l'ignorance, L'hydre infaite, et hideuse, et noire de venin, Conduisant bravement ceste conqueste a fin, Tu mez de plus en plus ta gloire en aparence. Tu fais ceste faveur aux escriteurs Toscans, D'esclarcir les escris de ceux qui plus scavanz Ont de toy cest honneur, qu'il te plaist les traduire. Quand Guicciardin verra ceste traduction, Il te voudra traduire, ainsi pourra ton nom, Aussi bien qu'en la France en l'Itale reluire ${ }^{42}$.

Comme en témoigne ce jeu de renversement des autorités, l'auteur ne dispose pas de l'autorité que l'époque romantique lui prêtera rétrospectivement ; parler de l'« émergence d'une conscience littéraire »semble plus approprié 43 . Sa participation à l'entreprise éditoriale - quand la publication se fait du vivant de l'auteur, ce qui quantitativement ne représente qu'une part restreinte des publications à la Renaissance va progressivement rendre possible la conquête de son autorité44. Non pas « défigurée », car non encore conquise, son autorité commence toutefois à être « figurée », c'est-à-dire représentée.

L'évolution de la présentation matérielle de l'ouvrage contribue en effet à afficher dans l'espace du livre la singularité de l'auteur (celle de son nom, de sa devise, de la langue qu'il choisit d'employer, etc.). Ainsi la pratique du portrait d'auteur passe-telle d'une représentation conventionnelle, renvoyant davantage à une instance, à une représentation plus individualisée et plus fidèle à la réalité 45 . Cette évolution rend bien compte des progrès de la gravure sur bois puis sur cuivre, et de la place nouvelle que prend l'image au sein du livre illustré au XVI ${ }^{e}$ siècle, dès lors que s'affinent les procédés de gravure et que se développe le marché de l'image imprimée 46 . Toutefois, dans le même temps, une telle évolution contribue à donner un visage à l'auteur, à le doter de traits singuliers distincts des attributs stéréotypés hérités de la tradition manuscrite

42. Lodovico Guicciardini, Les Heures de recreation et apres-disnées, Paris, Jean Ruelle, 1571, $\mathrm{f}^{\circ} \mathrm{Dd} 8 \mathrm{r}^{\circ}$.

43. On renverra notamment à l'article de François Rigolot, « Clément Marot et l'émergence de la conscience littéraire à la Renaissance », dans Gérard Defaux (dir.), La Génération Marot. Poètes français et néo-latins (1515-1550), Paris, Champion, 1997, p. 21-34.

44. Le cas de Clément Marot, lui-même éditeur des écrits de François Villon et de son père Jean Marot, est à cet égard précieux. Voir à ce sujet les travaux de Guillaume Berthon et notamment L'Intention du poète. Clément Marot « autheur », Paris, Classiques Garnier, 2014, qui analyse l'implication de l'auteur dans la publication de ses œuvres.

45. Nous renvoyons sur ce sujet au mémoire de Laure Bourgeaux, Bénédicte Jarry et Sophie Renaudin, «L'apparition du portrait gravé dans le livre au XVIe siècle », Enssib, 2005. Voir également le catalogue de l'exposition « Portraits d'écrivains : la représentation de l'auteur dans les manuscrits et les imprimés du Moyen Âge et de la première Renaissance, Poitiers, Médiathèque François-Mitterrand, 23 juillet-26 octobre 2002 », Paris, Fédération française de coopération entre bibliothèques, 2002.

46. Voir Michel Pastoureau, «L'illustration du livre : comprendre ou rêver ? », dans Roger Chartier et Henri-Jean Martin (dir.), Histoire de l'édition française, t. I, op. cit., p. 501-529. 
médiévale. De la même manière, si les pièces liminaires, signées de l'imprimeur, du traducteur, des connaissances et proches de l'auteur, contribuent à distribuer la parole entre différents énonciateurs, elles tendent à faire converger les discours en direction de l'auteur dont il est généralement fait l'éloge, l'inscrivant dans un espace énonciatif où d'autres auteurs trouvent à s'exprimer. Comme le rappelle Philippe Desan,

[1]es épîtres, poèmes dédicatoires, et autres pièces de ce genre annoncent et décorent à la fois le texte qui suit ; ils le présentent synthétiquement, établissent un lien direct - souvent biographique entre l'auteur et l'objet de sa production ; ils s'efforcent aussi d'expliquer - et donc de contrôler le texte, ou du moins de guider le lecteur au fil des pages afin que celui-ci ne s'écarte pas trop de l'effet recherché par l'intention originale de l'auteur. Le péritexte peut même éventuellement servir de plate-forme ou de vignette publicitaire réservée à d'autres auteurs à qui on offre cet espace pour publier quelques-uns de leurs sonnets ou dizains qui, à leur tour paraîtront plus tard dans leurs propres recueils et œuvres 47 .

Tout ce dispositif éditorial, qu'il soit discursif ou typo-iconographique, se surajoute donc au texte initial et non achevé de l'auteur, produisant un discours polyphonique. Mais il affirme dans le même temps la place singulière qu'y tient l'auteur en tant qu'instance désormais dotée d'une autorité distincte ${ }^{48}$. Celui-ci s'incarne en une « figure d'auteur ${ }^{49}$ » et ce, au moment même où l'exploration de nouvelles techniques imprimées ouvre encore à ses collaborateurs éditoriaux de nouveaux espaces d'innovation. En ce sens, l'autorité de l'auteur, plus que « conquise », individuellement, ou " défigurée » par le partage de la paternité littéraire, est « promue » collectivement. Si la création de l'œuvre, en tant que texte mis en livre et destiné, par l'entremise de l'appareil éditorial, à un public, est bien collaborative, l'acte de publication tend à en compenser les traces et signatures par la mise en avant d'une figure d'auteur, chez qui se développe progressivement la conscience d'une autorité et de droits auxquels il peut prétendre. Aussi peut-on considérer que l'efficacité d'une telle création collaborative a pu contribuer, en favorisant la promotion de la figure de l'auteur, à l'avènement de ce que Bénichou appellera le «sacre de l'écrivain ».

La perspective qu'offre une réflexion sur les pratiques collectives d'élaboration de l'œuvre à la Renaissance et son impact sur l'autorité - effective et en représentation - de l'auteur met au jour un certain nombre des spécificités de la création littéraire sous l'Ancien Régime. La production littéraire, dans le processus même de constitution du texte jusqu'à

47. Philippe Desan, L'Imaginaire économique de la Renaissance, Paris, PUPS, 2002, p. 144.

48. On remarquera par exemple que dans les premières Bibliothèques imprimées, comme celle de Du Verdier, les ouvrages commencent à être classés par noms d'auteur à l'intérieur des catégories qui les rangent d'abord par matières.

49. Cette première représentation préfigure ce qui deviendra une véritable « image d'auteur » avec l'entrée dans l'ère des médias modernes. Sur la notion d'image d'auteur, voir l'article de Dominique Maingueneau, « Auteur et image d'auteur en analyse du discours », Argumentation et analyse du discours [en ligne], n 3, 2009, <http://aad. revues.org/660>. 
son aboutissement ${ }^{50}$, ne peut se penser, à cette époque, sans le dispositif de médiation qui le conditionne, voire le détermine ${ }^{51}$. Ainsi, l'espace public de l'imprimé, loin d'être considéré comme un espace de fixation définitive du texte, apparaît comme le lieu de sa " mise à l'épreuve », une « mise en situation » de communication littéraire qui en relance en quelque sorte la performance ${ }^{52}$. Au sein de ce dispositif, l'auteur devient une figure nécessaire, et son auctorialité mise en avant pour reléguer dans l'ombre les manipulations éditoriales, tout comme le peintre, par la dernière couche qu'il dépose sur la toile, camoufle ses premiers croquis et repentirs ou signe encore le tableau en partie préparé par ses élèves des quelques traits qui assureront alors le succès de l'œuvre et son attribution.

50. En privilégiant une réflexion sur les présupposés et implications de la notion de « création collaborative » mise en regard de celle de « création d'atelier » à la Renaissance, nous n'avons pas abordé les cas pourtant fort intéressants de la correction sous presse effectuée par l'auteur ou par son secrétaire, du rafraîchissement textuel d'un écrit dans l'atelier de l'imprimeur par un remanieur, des procédés de montage intertextuel engagé par l'éditeur lui-même qui conçoit une œuvre inédite par regroupement de pièces déjà imprimées ou inédites, etc. Toutes ces situations éditoriales rendent compte en pratique des modalités de réalisation de cette création collaborative qui touchent le texte lui-même. L'Histoire de l'édition française (op. cit.) présente de nombreux cas qui illustrent ces phénomènes.

51. Nous renvoyons à cet égard aux différents travaux sur la sociabilité littéraire et notamment l'ouvrage de Christian Jouhaud et Alain Viala, qui y intègre les milieux éditoriaux : De la publication. Entre Renaissance et Lumières, Paris, Fayard, 2002.

52. Voir Michel Jeanneret, « Chantiers de la Renaissance. Les variations de l'imprimé au Xvie siècle », Genesis, $\mathrm{n}^{\circ} 6$, « Enjeux critiques », 1994, p. 25-45. 
AnNe RÉACH-Ngô, maître de conférences à l'Université de Haute-Alsace et membre de l'Institut universitaire de France, s'intéresse à la participation des milieux éditoriaux à la constitution du champ littéraire au Xvie siècle (L'Écriture éditoriale à la Renaissance. Genèse et promotion du récit sentimental français (1530-1560), Droz, 2013). Elle a (co)dirigé plusieurs ouvrages collectifs portant sur les pratiques éditoriales sous l'Ancien Régime : Le Discours du Livre (Garnier, 2011); Créations d'atelier. L'éditeur et la fabrique de l' euvre à la Renaissance (Garnier, 2014), ou sur les mutations du livre et de l'écrit en diachronie : L'Acte éditorial. Publier à la Renaissance et aujourd' hui (Garnier, 2010) ; L'Écrit à l'épreuve des médias (Garnier, 2012) ; Le Livre, produit culturel ? (Ôrizons, 2012). Elle a également coordonné la partie thématique du numéro 10 de la revue Seizième Siècle sur la question des «Genèses éditoriales » (2014). Ses travaux de recherche actuels portent sur la vogue éditoriale des « Trésors imprimés en langue vernaculaire ».

anne.reachngo@yahoo.fr

Résumés

\section{Du texte au livre, et retour : la production littéraire à la Renaissance, une création collaborative ?}

Si l'on assiste au XVIe siècle à l'émergence d'une première conscience littéraire de l'écrivain, cela tient à l'apparition progressive d'un statut symbolique et juridique de l'auteur. L'analyse de la répartition des tâches et des responsabilités lors de l'élaboration de l'œuvre littéraire dans l'atelier de l'imprimeur invite toutefois à interroger les présupposés, enjeux et implications qui autorisent à parler de " création collaborative ». Le dispositif de présentation de l'œuvre imprimée porte bien les traces d'un partage de la paternité littéraire, mais l'on voudrait montrer que la rhétorique discursive et typo-iconographique qui l'accompagne, loin de mettre à bas l'autorité naissante de l'auteur, tend au contraire à en assurer la promotion, au sein même d'un dispositif collectif qui relève d'une « création d'atelier ».

In the $16^{\text {th }}$ century, with the gradual development of the writer's symbolic and legal status, emerges the first literary awareness of the author. The analysis of the distribution of tasks and responsibilities involved in the making of a literary work in the printer's workshop, calls us to question the assumptions, issues and implications that allowing us to speak of "collaborative creation". The system of presentation of the printed work does indeed bear the traces of a shared literary paternity, but we would like to show that the discursive and typo-iconographic rhetoric that goes with it, far from negating the writer's nascent authority, tends on the contrary to ensure its promotion, at the very heart of the collective system which is a "workshop creation".

Wenn man dem 16. Jahrhundert das Emporkommen eines ersten literarischen Selbstbewusstseins des Schriftstellers zuschreibt, so bezieht sich dies auf das allmähliche Auftauchen eines eigenen symbolischen und rechtlichen Status des Autors. Die Analyse der Aufteilung der Aufgaben und Verantwortlichkeiten, die mit der Ausarbeitung eines literarischen Werkes im Atelier des Druckers zusammenhängen, lädt jedenfalls dazu ein, die Voraussetzungen, Probleme und Implikationen zu hinterfragen, die dazu führen, dass man von einer „kollaborativen Schöpfung“ spricht. Das Dispositiv der Präsentation eines Druckwerks führt zu Spuren einer Funktionsaufteilung innerhalb der literarischen Urheberschaft. Aber wir versuchen zu zeigen, dass die diskursive und die typographisch-ikonographische Rhetorik, die damit einhergeht - weit entfernt davon, die sich herausbildende Autorität des Autors zu untergraben -, im Gegenteil dazu führt, seine Autorität zu festigen, nämlich im kollektiven Dispositiv, das von einer „Atelier-Struktur“ abhängt.
Nel XVI secolo, l'affiorare di una coscienza letteraria dello scrittore è legato all'apparizione progressiva di uno statuto simbolico e giuridico dell'autore. L'analisi della ripartizione di compiti e responsabilità durante l'elaborazione dell'opera letteraria nella bottega del tipografo ci invita, tuttavia, a interrogare i presupposti e le implicazioni che ci autorizzano a parlare di "creazione collaborativa". Il meccanismo di presentazione dell'opera stampata porta certo le tracce di una condivisione della paternità letteraria, ma noi vorremmo dimostrare che la retorica discorsiva e tipografico-iconografica che lo accompagna, lungi dal demolire la nascente "autorità" dell'autore, tende al contrario ad assicurarne la promozione, nel seno stesso di un dispositivo collettivo caratteristico di una "creazione di bottega".

Se a partir do século XVI assistimos à emergência de uma primeira consciência literária do escritor, isso deve-se ao progressivo aparecimento do estatuto simbólico e jurídico do autor. A análise da distribuição de tarefas e responsabilidades na produção da obra literária dentro da oficina do impressor levam a questionar os pressupostos, desafios e implicações que permitem falar de "criação colaborativa". O dispositivo de apresentação da obra impressa revela as marcas de uma paternidade literária partilhada. Em todo o caso, a retórica discursiva e tipoiconográfica que o acompanha, longe de pôr em causa a autoridade nascente do autor, tende em vez disso a promovê-la, ainda que dentro de um dispositivo coletivo com características de "criação oficinal".

La emergencia, durante el siglo XVI, de una primera conciencia literaria del escritor resulta del surgimiento progresivo de un estatus simbólico y jurídico del autor. El análisis de la repartición de tareas y responsabilidades durante la elaboración de la obra literaria en el taller del impresor nos incita a interrogarnos acerca de los presupuestos, las estrategias y las implicaciones que nos autorizan a hablar de "creación colaborativa". El dispositivo de presentación de la obra impresa exhibe las huellas de una repartición de la paternidad literaria, pero es necesario indicar como la retórica discursiva y tipo-iconográfica, lejos de atentar contra la autoridad naciente del autor, tiende, por el contrario, a asegurar su promoción, en el seno mismo de un dispositivo colectivo que da cuenta de una "creación de taller". 